\title{
Design and evaluation of optics student competitions and contests for maximal educational value
}

\section{Stephen Pompea, Constance Walker}

Stephen M. Pompea, Constance E. Walker, "Design and evaluation of optics student competitions and contests for maximal educational value," Proc. SPIE 9664, Ninth International Topical Meeting on Education and Training in Optics and Photonics, 96641I (24 October 2005); doi: 10.1117/12.2207731

SPIE Event: Ninth International Topical Meeting on Education and Training in Optics and Photonics, 2005, Marseille, France 


\title{
Ref ETOP068
}

\section{Design and Evaluation of Optics Student Competitions and Contests for Maximal Educational Value}

\author{
Stephen M. Pompea ${ }^{1}$ and Constance E. Walker ${ }^{2}$ \\ ${ }^{1}$ National Optical Astronomy Observatory, Tucson, Arizona, 85719 USA \\ ${ }^{2}$ Carolyn Peruta University of Arizona, Tucson, Arizona, 85721, USA
}

\begin{abstract}
The National Science Foundation-funded project "Hands-On Optics: Making an Impact with Light" is developing six educational modules designed for an informal museum, science center, or other "free-choice" audience for use by students aged 10-17. The project is a joint project of the SPIE-The International Society for Optical Engineering, the Optical Society of America, and the National Optical Astronomy Observatory. The Project Principal Investigator is Dr. Anthony Johnson, the Director of the Center for Advanced Studies in Photonics Research (CASPR) at the University of Maryland, Baltimore County. The Hands-On Optics program also has designed and sponsored student competitions that immerse students in a problem-solving environment. We describe how two student optics competitions we have designed can be used to motivate student work in optics. In the "Telescope" competition, students build and test a Newtonian-style reflecting telescope. In the "Hit the Target" competition, students reflect light off of mirrors and through an obstacle course in order to see how close they can come to hitting a target.
\end{abstract}

\section{Key Words}

Optics education, reflection, telescope, laser, engineering design, problem-solving

\section{Summary \\ Introduction}

The Hands-On Optics project is developing six educational modules for national distribution that address the basics principles of optics. In order to extend the project's reach, the HandsOn Optics program also has designed and sponsored student competitions which immerse students in a problem-solving environment. We describe how a number of optics student competitions we have designed can be used to motivate student work in optics. Two competitions have been designed to date. In the "Telescope" competition, students build and test a Newtonian-style reflecting telescope. In the "Hit the Target" competition, students align mirrors in order to send laser light through an obstacle course in order to see how close they can come to hitting a target. The competitions represent an extension of the educational modules of the program and are conducted by students who have completed related modules.

The six Hands-On Optics modules cover concepts such as simple reflection from mirrors and the law of reflection, reflection from multiple mirrors and kaleidoscopes, formation of images and construction of a refracting telescope, polarization and how birefringence can be used to measure stress, properties of infrared and ultraviolet light and how they interact with materials, and communication over light beams.

Each module is designed with an understanding of student misconceptions and naïve theories about optics, a sense of how math can be integrated into the science of optics, and with an appreciation of how teachers can best acquire basic optics concepts. A key part of the program is the intense involvement of optics industry volunteers as resource agents with 
strong content and science process knowledge. These volunteers assist the educator in understanding optics concepts and the techniques used by optical scientists and engineers.

\section{Rationale for competitions}

To provide a means of allowing students to apply the knowledge they have gained in the Hands-On Optics program, the project is designing a series of competitions that allow students to work in a more open-ended, inquiry-oriented way with the knowledge and equipment that they have been using. These competitions also provide a way to encourage the development of teamwork skills and the ability to understand the problem, divide up tasks, and to best utilize the individual skills of team members. One of the key audiences for the Hands-On Optics project is the Mathematics, Engineering, Science Achievement (MESA) program, an after-school program found primarily in the western United States. MESA emphasize students preparing for and participating in competitions on a local and statewide level. The creation of optics competitions allows MESA students to continue their involvement in optics beyond their initial work with the optics kits.

\section{Telescope competition}

A telescope construction competition was chosen in order to combine the key concepts of the first three modules. In module one students learn about reflection from specular and diffuse surfaces. In module two, they learn about multiple reflections from plane mirrors. In module three, they learn about image formation by lens and mirrors, and they construct a simple refracting telescope. After constructing the telescope, they measure its magnification and test its resolving power.

A telescope competition has students determining the focal lengths of lenses and mirrors in

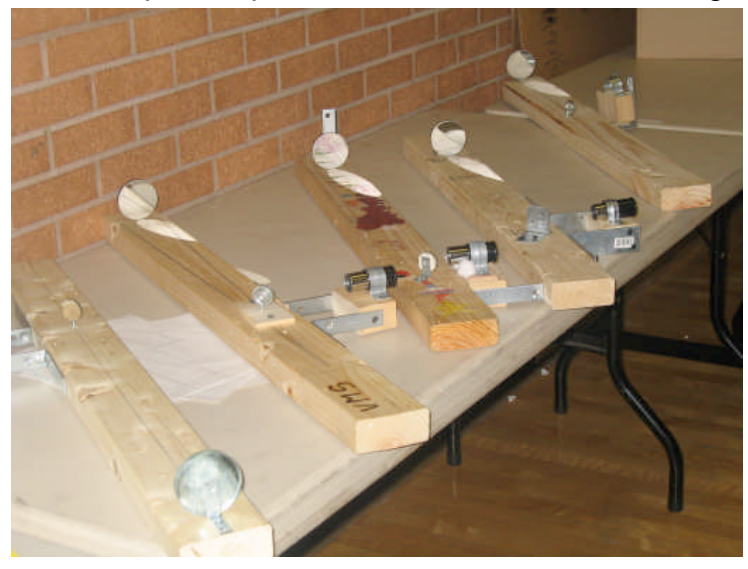
order to build a Newtonian-type reflecting telescope. The students are given instructions on the basic design of the telescope but must determine the focal lengths of the primary mirror (a three-inch $f 10$ spherical mirror) and eyepiece. With this information, the students can construct the telescope. After the telescope is built, the students align the telescope, and evaluate the resolution. The competition relies on the correct determination of the focal lengths and how well the teams build and align their telescopes. A resolution chart is also used to test the telescopes.

Teams are judged on the total system performance of the final telescope. If the telescope is built and aligned properly, the telescope will perform to its resolution limits. If the telescope is aligned improperly, significant changes in resolution will occur. Small changes in the placement of the flat diagonal mirror, or in the position of the eyepiece can result in significant changes in performance. Serious problems in alignment or placement of the mirrors will make the telescope inoperable.

The telescopes were also judged for their appearance and quality of construction as well as for their performance. Students were asked to draw the optical design and a ray trace of their system on a piece of paper. The photograph of several telescopes illustrates the simplicity of the telescopes. The primary mirror is glued to an L-bracket that is attached to a wooden 
board. The flat secondary mirror is attached to an eye-bolt screwed into the wood. Additional brackets hold the eyepiece. It was decided not to mount the telescopes in a tube. This needlessly complicated the design, increased the expense of the kit, and obscured the basic design. All of the optics components were obtained as surplus optics and all of the other parts were obtained at a hardware store.

Because of the lack of a mount for the telescopes, they were tested by laying them flat on a table and positioning a target about 25 feet away. In this orientation, the resolution of the telescope in viewing the target could be easily determined. Various targets were used, including type printed in different font sizes, official resolution targets, and dollar bills. The use of money was an effective target, since money typically has a wide variety of type sizes and details at various scale lengths. This competition has been used for two years in Arizona with MESA students and has proved popular with students in the 10-17 years age range.

\section{"Hit the target" competition}

In a laser reflection competition, students who have completed module one are under time pressure to position and align mirrors to order to have a laser beam hit a target. The students are evaluated on how closely the beam comes to the target. In this competition, the students are not allowed to turn on the laser beam until after all alignment efforts have been conducted. They must use protractors, strings, and other alignment aids but are not allowed to turn the laser on until they are ready to be judged. This competition tests the students' comprehension of the law of reflection. Students are not specifically taught alignment techniques but are encouraged to measure angles relative to the mirror surface normal in order to predict where the beam will go. The score for each student team is determined by the number of different tries needed to hit the target, and by how close the laser beam comes to the center of the target, which is typically a few square inches in area and placed on a wall near ground level.

\section{The role of authentic assessment}

In addition to their motivational value, the competitions are also especially useful as a form of assessment for the project modules. As in the modules, students work in small groups when they compete. The competitions provide an authentic assessment of the students and the skills they have developed. This form of assessment, in contrast to more objective tests on paper, best resembles how industrial engineering teams are evaluated. Performance in the competition is a solid measure of student comprehension of the modules and their ability to apply these concepts in new situations.

\section{Conclusion and lessons learned}

These competitions have been tested at a variety of grade levels and are motivational to the optics students. Surprisingly, older students (ages 15-18) do not do appreciably better in telescope construction than younger students (ages 11-14). The educational effectiveness of the competitions is strengthened through orientation workshops given to teachers on the competition. In these competitions, the use of college science students as judges has been found to be most effective. These students not only do an excellent job of judging the competitions but also serve as role models for younger students involved in the competitions. As in most competitions, simple and well-written rules and scoring rubrics are essential.

\section{Contact Information}

Dr Stephen M. Pompea, Hands-On Project Co-Investigator

Manager of Science Education, and Astronomer, National Optical Astronomy Observatory, Tucson Arizona 85719 USA. Pompea is also Education Co-Chair of the Arizona Optics Industry Association and Adjunct Faculty, University of Arizona. Voice 520.318.8285 Email spompea@noao.edu

The Hands On Optics Project is funded by the National Science Foundation ISE program. NOAO is operated by the Association of Universities for Research in Astronomy (AURA), Inc. under cooperative agreement with the National Science Foundation 